\title{
Early diagnosis to enable early treatment of pre-osteoarthritis
}

\author{
Constance R Chu ${ }^{* 1,2}$, Ashley A Williams', Christian H Coyle ${ }^{1}$ and Megan E Bowers'
}

\begin{abstract}
Osteoarthritis is a prevalent and disabling disease affecting an increasingly large swathe of the world population. While clinical osteoarthritis is a late-stage condition for which disease-modifying opportunities are limited, osteoarthritis typically develops over decades, offering a long window of time to potentially alter its course. The etiology of osteoarthritis is multifactorial, showing strong associations with highly modifiable risk factors of mechanical overload, obesity and joint injury. As such, characterization of pre-osteoarthritic disease states will be critical to support a paradigm shift from palliation of late disease towards prevention, through early diagnosis and early treatment of joint injury and degeneration to reduce osteoarthritis risk. Joint trauma accelerates development of osteoarthritis from a known point in time. Human joint injury cohorts therefore provide a unique opportunity for evaluation of pre-osteoarthritic conditions and potential interventions from the earliest stages of degeneration. This review focuses on recent advances in imaging and biochemical biomarkers suitable for characterization of the pre-osteoarthritic joint as well as implications for development of effective early treatment strategies.
\end{abstract}

\section{Introduction}

Osteoarthritis (OA), a leading cause of morbidity and disability, carries high socioeconomic costs. In 2004 arthritis was estimated to cost the United States $\$ 336$ billion, or $3 \%$ of gross domestic product [1]. OA is by far the most common form of arthritis. With increasing obesity and age in the population, a massive rise in morbidity and costs attributed to OA is expected.

*Correspondence: chucr@upmc.edu

2Department of Orthopaedic Surgery, University of Pittsburgh, 3471 Fifth Avenue, Suite 911, Pittsburgh, PA 15213, USA

Full list of author information is available at the end of the article
While joint replacement is effective for treating end-stage OA in older people, evaluation of potential diseasemodifying treatments in populations meeting current clinical criteria for OA has had limited success. Early diagnosis and early treatment strategies in rheumatoid arthritis have reduced patient morbidity and associated costs in the past decade. New concepts for early diagnosis and treatment of pre-osteoarthritic conditions may similarly improve outcomes and reduce disability and costs for OA, the most prevalent form of arthritis.

\section{Pre-osteoarthritis is a modifiable disease process}

Epidemiological and genetic studies of OA indicate that many pre-OA disease states can be modified. While OA can affect any joint, substantial disability is attributed to OA of the weight-bearing joints, primarily the hip and knee. OA is a multifactorial decades-long process reflecting a complex interplay between intrinsic and extrinsic factors. While there is evidence for heritability of OA $[2,3]$, the polygenic nature of the disease with multiple genes contributing small effects has made it difficult to identify the genetic etiologies of OA [4]. Genome-wide association studies have yielded few common genetic targets [5]. Whereas OA is the culmination of multivariate interactions between genetic, epigenetic, and environmental factors, extrinsic factors such as obesity, trauma, and joint loading patterns are known to heighten OA risk and to offer more definable targets for disease modification. The systematic study of large cohorts at increased risk for accelerated OA development therefore has potential not only to yield new disease-modifying treatments but to facilitate improved understanding of the complex interactions between genes and the environment in OA development [6].

\section{Extrinsic events such as joint trauma accelerate osteoarthritis development}

Post-traumatic OA illustrates the concept that modifiable extrinsic factors play a substantial role in OA development. Joint trauma such as intra-articular fracture, dislocations, anterior cruciate ligament tear (ACLT), and other injuries lead to rapid joint degeneration in a high proportion of patients $[7,8]$. Articular surface incongruity, 
joint instability, altered kinematics, articular cartilage injury, and other joint-tissue changes attributable to the traumatic event accelerate OA development. In a longterm prospective cohort study, young adults with knee injuries showed substantially increased risk for later development of osteoarthritis of the index knee [8]. Another study showed that roughly one-half of individuals with ACLTs or meniscus tears developed radiographic signs of OA 10 to 20 years after injury [9]. Since ACLT is most frequently sustained by teenagers and young adults, it can be considered the cause of premature knee $\mathrm{OA}$ in these patients - a devastating outcome with costly social and economic consequences.

\section{Joint injury cohorts enable characterization of pre- osteoarthritic processes from the earliest stages}

For the study of pre-OA conditions, joint injury cohorts offer the potential to study, characterize, and modify the disease process from its earliest stages. A recent American Orthopaedic Society for Sports Medicine/ National Institutes of Health U-13 multidisciplinary conference focused on post-joint injury OA described advantages for studying meniscus-injured and anterior cruciate ligament (ACL)-injured cohorts [6]. These cohorts represent populations that do not meet the classic radiographic or clinical criteria for OA [10]. Rather, subjects have joint pathologies placing them at risk for accelerated OA development. These populations offer opportunities to define and treat pre-OA conditions. The acute ACL and meniscal injury populations are well suited for bench-to-bedside translational studies of new treatment strategies because they are more similar to popular animal models of pre-clinical OA such as ACL transection and meniscus injury than the more heterogeneous older human cohorts with established multi-site OA traditionally used to evaluate potential disease-modifying treatments [6].

Defining and characterizing pre-OA in its earliest stages is crucial not only for understanding the disease process, but also for identifying potential diseasemodifying treatments and evaluating their effectiveness. Early diagnosis will permit earlier treatment to modify the course of this disease. This review will focus on recent advances in imaging and biochemical biomarkers suitable for characterization of the pre-osteoarthritic joint.

\section{Imaging of the pre-osteoarthritic joint}

Radiographs have been used for more than a century to assist diagnosis of bone and joint disorders. The radiographic hallmarks of structural changes in classic OA include osteophyte formation, joint-space narrowing, subchondral bone thickening, and cyst formation. Clinical criteria of pain, stiffness, and age are poorly correlated to radiographic findings. Radiographic joint-space narrowing, an indirect and highly insensitive measure of articular cartilage integrity, is the current standard by which clinical efficacy of disease-modifying therapies for OA are measured [11]. Injuries to menisci, articular cartilage, and other joint structures readily apparent at arthroscopy or arthrotomy, as well as stress fractures and even early avascular necrosis, are not detectable by standard radiographic examination. Efforts to develop and assess disease-modifying treatments for $\mathrm{OA}$ have been hampered by the insensitivity of these traditional clinical and radiographic measures to joint-tissue health prior to the development of end-stage disease.

Advances in optical imaging and magnetic resonance imaging (MRI) have permitted direct imaging of joint tissues. Arthroscopy permits direct visualization and palpation of intra-articular structures. The sensitivity of MRI for soft tissue assessments and bone marrow changes exceeds that of conventional radiographs. While MRI lacks the tactile and dynamic examination capabilities of arthroscopy, it permits noninvasive and quantitative assessments of joint-tissue structure. As such, MRI offers many advantages for diagnosis and staging of preOA joints. While assessment of thin and geometrically complex tissues such as articular cartilage remains challenging for MRI due to low resolution, newer techniques of quantitative, volumetric, and physiologic MRI show promise. Optical coherence tomography (OCT), a new high-resolution imaging technology permitting microscopic cross-sectional imaging of soft tissues in near real time, has been used arthroscopically as a translational research tool to assist in evaluating quantitative MRI of articular cartilage [12]. Finally, measuring joint synovitis reflects increasing recognition of an inflammatory component in pre-OA conditions and in OA pathogenesis.

\section{Arthroscopy and arthroscopic optical coherence tomography}

Although the first known report of knee arthroscopy was presented in 1912 [13], only after key advances in fiberoptic and video technologies occurred in the 1970s and 1980s did arthroscopy became widely used for direct visualization, examination, and treatment of intra-articular soft tissues. Radiographically invisible pathologies such as meniscal tears, articular cartilage lesions, and cruciate ligament tears can be readily seen and palpated by arthroscopy. While subjective, the surgeon's assessment of articular cartilage softening represents the earliest detectable clinical sign of pre-OA changes to articular cartilage known as chondromalacia or chondrosis (Figure 1) [14].

Conventional arthroscopy has continued fundamentally unchanged as a high-resolution surface imaging technology for which subsurface structure is inferred through 


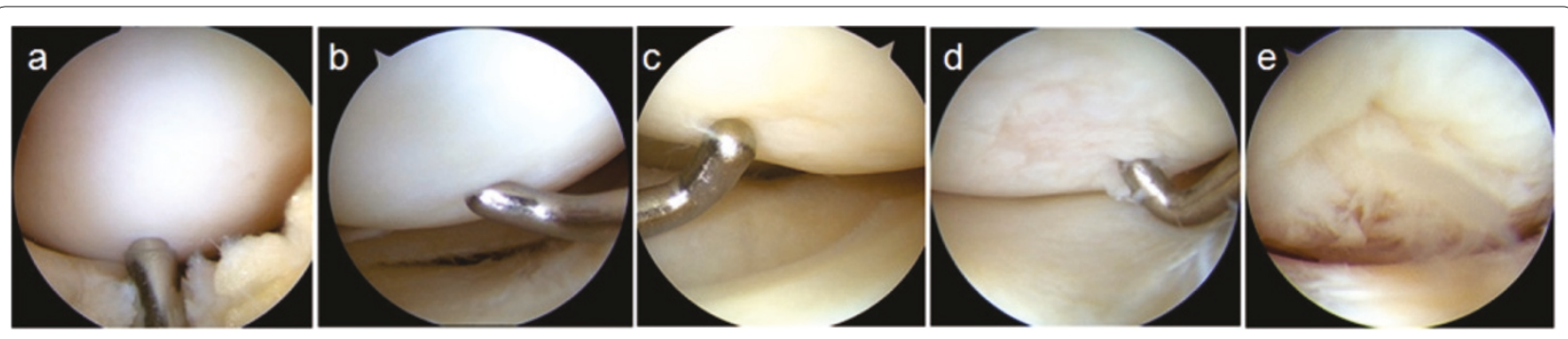

Figure 1. Chondropathy and the modified Outerbridge system. Sample arthroscopic images demonstrating different grades of the modified Outerbridge scoring system. (a) Grade 0: smooth, firm articular cartilage. (b) Grade 1: articular cartilage is surface-intact, but softened. (c) Grade 2: articular cartilage with a damaged surface $<50 \%$ of tissue depth. (d) Grade 3: articular cartilage with a damaged surface $>50 \%$ of tissue depth. (e) Grade 4: articular cartilage with full-thickness tissue disruption extending to the subchondral bone.

subjective palpation. In the past decade, OCT has been translated from bench to bedside to perform microscopic cross-sectional imaging of articular cartilage [12,15]. OCT provides high-resolution ( 4 to $20 \mu \mathrm{m}$ ) crosssectional imaging of articular cartilage that is comparable with low-power histology. Furthermore, OCT imaging does not involve ionizing radiation or risks of radiation exposure. Polarization-sensitive OCT detects changes in the polarization state of backscattered light and could be used to detect subsurface cartilage degeneration, because its associated collagen disorganization results in loss of polarization sensitivity [16]. This loss of birefringence has been seen in human cartilage both in vitro and in vivo [17].

For the characterization of pre-osteoarthritic clinical cohorts, OCT may complement conventional arthroscopy and quantitative magnetic resonance imaging (qMRI). A recent review discusses advantages of arthroscopic OCT for evaluation of subsurface cartilage abnormalities that may elude conventional arthroscopic surface imaging and MRI [18]. Comparison of OCT with MRI T2 mapping and arthroscopy in human subjects with degenerative meniscal tears revealed that OCT can detect subsurface cartilage degeneration and is a useful translational clinical research tool. While no correlation was found between MRI T2 mapping and arthroscopy, OCT correlated with both techniques [12]. This was probably because OCT shared characteristics of high resolution with arthroscopy and of cross-sectional quantitative data with MRI T2 mapping. OCT also detected early changes in articular cartilage that otherwise appears normal by gross inspection in bovine osteochondral cores following impact injury [19]. In evaluating the articular cartilage of human subjects following ACL injury, OCT shows microscopic subsurface changes suggestive of collagen matrix disruption (Figure 2).

\section{Magnetic resonance imaging}

MRI permits direct imaging of soft tissues. For evaluation of pre-OA conditions, conventional MRI permits morphological assessment of cartilage and meniscus, as well as other intra-articular and peri-articular soft tissues. Bone marrow changes are also well seen by conventional MRI. Subchondral bone attrition can be seen with MRI during early OA, but its significance is still poorly understood. A recent study shows that regional subchondral bone attrition is strongly associated with cartilage loss in the same region and may indicate areas of the knee joint experiencing great compressive stress [20]. Because OA development involves the whole joint, several semiquantitative grading scales for standard MRI have been developed, including the Whole Organ MRI score and the Boston Leeds Osteoarthritis Knee Score [21].

qMRI includes volumetric measurement and physiologic MRI. Advances in qMRI include development of high-field (3T) three-dimensional sequences such as double-echo steady state and fast low-angle shot [22]. These sequences can detect cartilage volume and thickness changes of only a few percent. Knee cartilage thickness losses of $<1 \%$ over 1 year have been identified in longitudinal volumetric assessments of $3 \mathrm{~T}$ double-echo steady-state data acquired from at-risk subjects with preradiographic OA or subjects with mild disease (KellgrenLawrence score 1) enrolled in the Osteoarthritis Initiative study [23].

\section{Physiologic quantitative MRI informs on subsurface structure and composition}

Subsurface alterations to the biomolecular integrity of articular cartilage and menisci may represent the earliest structural changes in pre-OA conditions, but they cannot be reliably detected by clinical radiography or conventional MRI [24]. Changes within the meniscus and deep layers of cartilage, where highly organized collagen fibrils restrict proton mobility and cause rapid relaxation of the MRI signal, are particularly challenging to measure by conventional imaging techniques. Several reviews of qMRI techniques that address this challenge are already available [25]. This section will focus on qMRI evaluation 

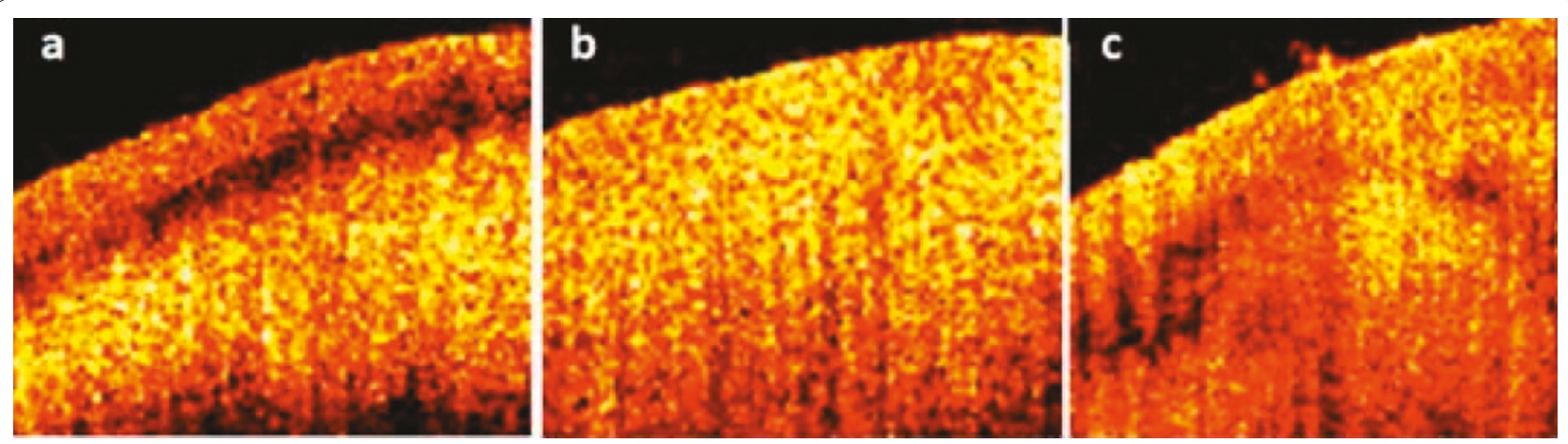

Figure 2. Optical coherence tomography. Optical coherence tomography (OCT) permits cross-sectional imaging of articular cartilage at resolutions comparable with low-power histology. (a) Arthroscopically firm and surface-intact cartilage shows OCT form birefringence. (b) Loss of birefringent banding on OCT correlates to softened cartilage. (c) Subsurface voids and fissures can be visualized with OCT.

of changes to articular cartilage and menisci in pre-OA human subjects.

\section{Physiologic quantitative MRI}

The primary components of articular cartilage and menisci are water, type II collagen, and proteoglycans. The goals of physiologic qMRI are to detect biochemical, spatial, and relational changes of these components through quantifying MRI signal changes. While multiple technologies are under active investigation - including, but not limited to, sodium MRI for evaluation of matrix proteoglycans and diffusion tensor MRI to measure tissue anisotropy - the primary physiologic qMRI techniques studied clinically in pre-OA cohorts include delayed gadolinium-enhanced magnetic resonance imaging of cartilage (dGEMRIC) [26-28], T2 and T1rho mapping [29-31], and ultrashort echo-time enhanced $\mathrm{T}^{*}$ * (UTE-T2*) mapping [32-34].

\section{Delayed gadolinium-enhanced magnetic resonance imaging of cartilage}

dGEMRIC is a technique used to map the relative concentration and spatial distribution of cartilage proteoglycan content [35]. The dGEMRIC index correlates with cartilage proteoglycan content and is decreased in OA, consistent with tissue glycosaminoglycan loss [35,36]. In early OA, lower dGEMRIC indices were seen in degenerated cartilage compartments compared with unaffected compartments [28]. Lower baseline dGEMRIC indices were found to predict $\mathrm{OA}$ disease presence in subjects with pre-radiographic OA [26]. The dGEMRIC index has also been shown to be potentially responsive to therapeutic interventions. Among meniscectomy subjects, examination of dGEMRIC before and after a 4-month change in exercise regimen found that subjects who increased their exercise level experienced a concurrent increase in the dGEMRIC index, indicating increased glycosaminoglycan content [27].

\section{T2 mapping}

T2 is sensitive to tissue hydration and collagen matrix organization. Previous studies have established that regional variations in cartilage matrix architecture result in significant variation in T2 values with articular cartilage depth and in cartilage across different regions of the knee [37,38]. Despite spatial variations, T2 values typically increase with cartilage degeneration. In early disease and in subjects at risk for developing OA, T2 has been shown to be focally elevated [39]. Longitudinal T2 has also been shown to change with activity. Light exercise in meniscectomy subjects at risk of developing OA was significantly associated with a decrease in T2 values compared with sedentary subjects or strenuous exercisers, suggesting a chondroprotective effect of lowintensity activities [29]. In females after meniscectomy, moderate and strenuous exercise (for example, running, cycling) was associated with elevated T2 values, suggesting cartilage degeneration with more intense exercise [29].

\section{T1rho mapping}

Like T2, T1rho is thought to be sensitive to tissue hydration and matrix macromolecular architecture [30]. Unlike T2, T1rho may also be sensitive to the proteoglycan content of the tissue; however, this point remains controversial [40]. Cartilage T1rho values generally tend to be higher in mild OA subjects compared with controls, but reports of a larger range and effect size for T1rho compared with $\mathrm{T} 2$ indicate that T1rho may be more sensitive to cartilage degeneration [30]. Further work is needed to illuminate the relative strengths and weaknesses of $\mathrm{T} 2$ and T1rho as qMRI techniques for evaluating early $\mathrm{OA}$.

\section{Ultrashort echo-time enhanced T2* mapping}

UTE-T2* imaging is a novel technique with the potential to measure short-T2 MRI signals from the meniscus and 
the deep layers of articular cartilage that are not well captured by conventional MRI or by standard T2 mapping. In vitro, UTE-T2* values have been shown to reflect collagen matrix integrity of human articular cartilage explants as determined by polarized light microscopy [41]. In vivo, UTE-T2* mapping is reproducible and shows quantitative subsurface changes to surface-intact articular cartilage and menisci in human subjects acutely following ACL injury (Figure 3).

\section{MRI of bone marrow, menisci and synovium in pre-osteoarthritis}

While both structural MRI and qMRI of thin, anisotropic, and geometrically complex articular cartilage remain a challenge even using high-field magnets, information concerning pathological changes to other joint structures implicated in joint degeneration such as bone marrow, menisci, and synovium is more readily obtained from conventional MRI. qMRI of menisci is a newer area of investigation that may also inform on subclinical meniscal injury and pathology.

\section{Bone marrow lesions}

Bone bruises and bone marrow lesions (BML) are commonly seen in traumatic joint injuries, in areas of cartilage loss, and following meniscal root tears. Because these injuries place affected joints at risk for accelerated OA, assessment of BML may be useful for evaluating preOA states. BML and bone marrow edema appear on MRI as areas of low signal intensity on T1-weighted images, and corresponding high signal intensity on T2-weighted images [42]. MRI is useful for assessing the presence and severity of BML, and for elucidating their relationships with early OA development.

Recent studies suggest that BML correlate spatially with cartilage degeneration and that BML may be an imaging marker for subsequent $\mathrm{OA}$ development. Correlation was found between joint effusions and BML [43]. BML have been associated with higher T1rho MRI values in articular cartilage adjacent to the bony edema [44]. Strong correlations were also found between BML and development of subchondral bone attrition [45].

\section{Meniscus}

Meniscus injury and degeneration play substantial roles in the development of knee OA. Loss of meniscus function, whether through trauma or degeneration, leads to accelerated joint degeneration. A recent study showed that complex meniscal tears were associated with elevated T2 relaxation times in the adjacent tibial cartilage [46]. Medial meniscus tear is common in the ACL-injured knee, and accelerated OA development is more common following combined ACLT and medial meniscus tear than isolated ACLT [6].
In recognition of the role of meniscus pathology in pre$\mathrm{OA}$, researchers have begun to examine the significance of intrasubstance MRI signal changes within the menisci, and whether these changes are associated with $\mathrm{OA}$ development [47]. Although menisci are typically assessed with structural MRI, semi-qMRI metrics may be useful for evaluating meniscal health, particularly in preOA. Using novel UTE-T2* mapping, menisci without evidence of tear by structural MRI or arthroscopy in humans with ACLT showed elevated T2*, suggesting subsurface matrix injury (Figure 3) [34].

Meniscal damage may trigger synovial activation, thereby affecting overall joint health. Evaluation of subjects without radiographic signs of OA in the Framingham and MOST studies showed that joint effusions were more prevalent in those patients with meniscal tears than in those without meniscal pathology [48]. This observation held even in knees with no MRI evidence of articular cartilage changes. These results further support the importance of meniscus assessment in identification of pre-osteoarthritic knees.

\section{Synovitis}

Assessment of synovitis may be important for the characterization and early treatment of pre-OA conditions. Synovitis has traditionally been considered a hallmark of inflammatory arthritis and less relevant to clinical OA. At total knee replacement for end-stage OA, however, synovitis was observed in $>90 \%$ of patients with nearly one-third showing severe inflammation, including histological evidence of lymphoid aggregates $[49,50]$. In human subjects with pre-OA conditions, synovitis is evident in synovial biopsies [51,52]. While both MRI and ultrasound have been used to noninvasively assess inflammation in osteoarthritic joints, MRI shows promise in systematic evaluation of synovitis along with evaluation of BML, menisci, and articular cartilage. A high prevalence of synovitis has been shown in people with knee OA, using both contrast-enhanced MRI and noncontrast MRI [53,54].

\section{Validation}

Imaging biomarkers, including OCT and physiologic MRI metrics, have been evaluated in a number of preclinical animal studies and in vitro studies with human cartilage. Strong correlations between OCT and histomorphometric analysis of cartilage repair tissue was established in rabbits [55], while split-line analyses and polarized light microscopy validated polarization-sensitive OCT detection of the three-dimensional structure of articular cartilage collagen matrix in equine fetlock joints [56]. MRI detection of cartilage lesions with dGEMRIC was shown to correspond to zones of mechanically intact cartilage with low proteoglycan content, as determined 

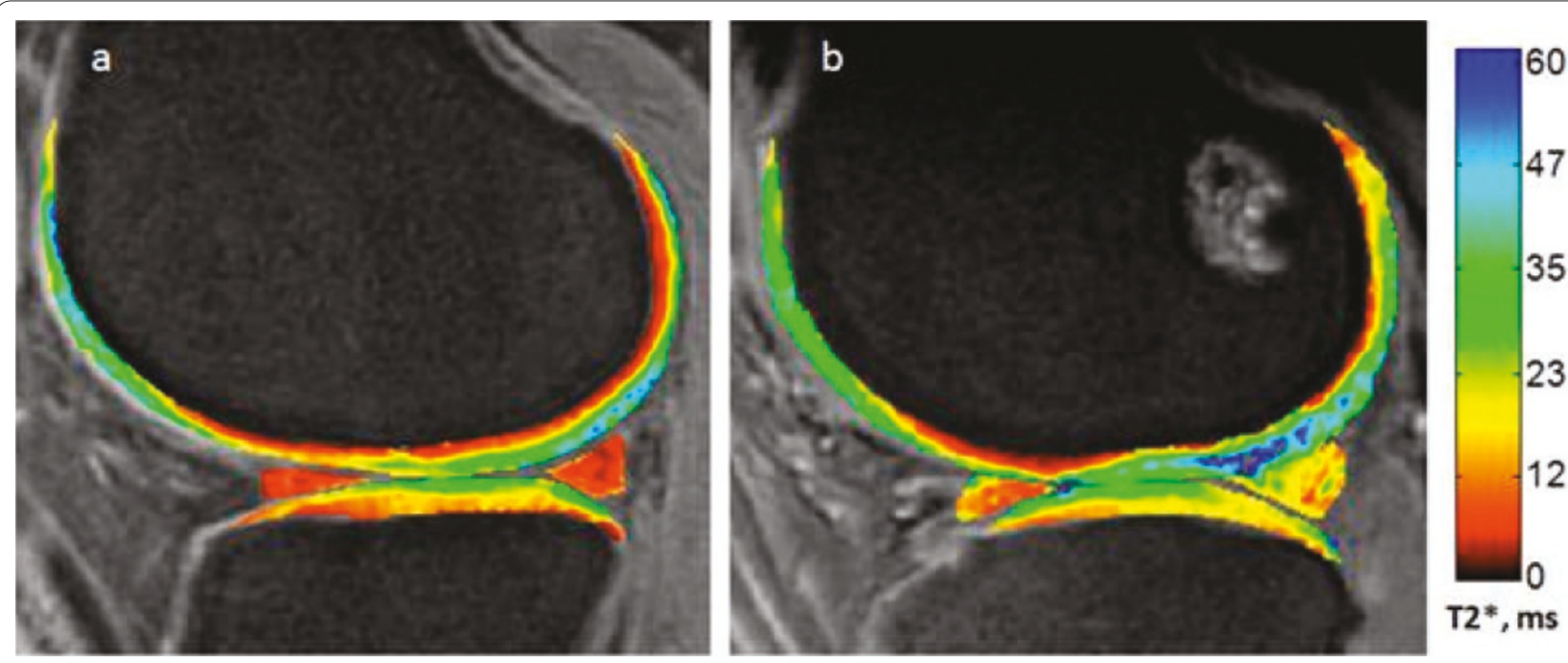

Figure 3. Ultrashort echo-time enhanced T2* maps of cartilage and meniscus show changes after knee injury. Ultrashort echo-time enhanced T2* (UTE-T2*) maps of articular cartilage and meniscus tissue show quantitative differences between (a) an uninjured asymptomatic subject and (b) an anterior cruciate ligament-injured subject with concomitant posterolateral meniscus pathology.

by azan-stained histologic sections obtained from human knees during total knee replacement. In both bovine and human explant studies, the dGEMRIC index was shown to correspond to histological assessments of proteoglycan content $[57,58]$. In a separate study, proteoglycan depletion due to trypsinization of bovine patellar cartilage was found to correspond to significant increases in T1rho [59]. Ex vivo studies have correlated OCT with $\mathrm{T} 2$ and polarized microscopy, $\mu$ MRI T2 with polarized microscopy, and UTE-T2* with collagen architecture as assessed by polarized microscopy [41,60,61]. Human cartilage removed during total knee replacement had high T2 and T1rho values and showed fibrillation by histology when compared with intact cartilage [57].

While human tissue and animal studies inform on imaging metrics, true validation requires human in vivo study. In a level I clinical diagnostic study, OCT was shown to correlate with arthroscopy while MRI T2 maps correlated with OCT [12]. The reliability of dGEMRIC as an indicator of proteoglycan content in deep cartilage when used clinically remains unclear, with several human clinical studies suggesting incomplete penetration of the contrast agent into deep articular cartilage $[58,62]$. In human studies, T1rho continues to prove sensitive to early OA, but more work is required to understand and demonstrate its specificity for molecular changes to the proteoglycan and/or collagen components of articular cartilage [40]. Similarly, because cartilage T2 reflects tissue hydration, collagen organization, and collagen fibril integrity, interpretation of T2 change in clinical studies remains difficult [40,63]. Recently, UTE-T2* mapping was shown to be potentially sensitive to subclinical meniscus damage in ACL-injured subjects
[34]. Because new imaging technologies are employed with the goal of detecting pathological changes that escape current clinical detection using technologies accepted as current clinical standards, longitudinal clinical studies will be needed to see whether potential imaging markers of early disease show predictive value.

\section{Biomarkers}

Metabolic changes in articular cartilage, synovium, and subchondral bone may represent the earliest measurable changes in pre-OA conditions. Metabolic perturbations are frequently considered reversible. As such, identification and validation of biomarkers for pre-OA states and at-risk joints may have wide application in clinical trials of new intervention strategies, in routine screening, as well as in activity-modification programs and returnto-play evaluations.

Characteristics of OA development include progressive loss of articular cartilage and increasing inflammation. These pathological states have been observed in various experimental animal models of OA, including meniscal destabilization and ACL transection. Advantages of studying biochemical biomarkers in animal models of OA include availability of biospecimens before injury and at precise intervals after injury where biomarker data can be correlated with structural information obtained from gross and histological evaluations. Biomarkers of cartilage matrix synthesis and degradation were found to be elevated during the early phases of experimental OA using the canine ACL transection model [64]. Structural changes to cartilage, bone, and menisci following ACLT and sham surgery in an ovine model were similarly shown to be highly correlated to inflammatory and 
degradative synovial biomarkers [65]. These and other preclinical studies of surgically induced joint injuries show promise in the use of biochemical biomarkers of joint-tissue turnover and inflammation for early detection and staging of pathological changes associated with later OA development.

In humans, however, the development and validation of biomarkers in traditional cohorts consisting of older individuals with clinical $\mathrm{OA}$ has been challenging, particularly due to variable disease states and multi-joint involvement. The challenge has been compounded by the insensitivity of traditional clinical outcome measures such as radiographs to structural and metabolic changes, as well as the poor correlation between clinical symptoms and radiographic changes, especially in early disease. Closing the apparent gap between the preclinical findings and clinical studies can be potentially effected through study of biomarkers in human clinical cohorts more similar to the animal models of surgical joint injury induced OA [6]. Specifically, systematic longitudinal evaluations of biochemical biomarkers following ACL and meniscus injury in human clinical cohorts that are also followed by structural and clinical outcome measures are needed. A subset of biomarkers derived from the degradation and formation of articular cartilage has been used for OA detection, and reviews of biomarkers in OA have been recently published [66,67]. As such, this review will focus on recent research assessing biomarkers for pre-OA conditions, especially following $\mathrm{ACL}$ and meniscus injuries.

\section{Inflammatory cytokines and mediators}

Inflammation is present following joint injury and its role is increasingly recognized in OA development. Inflammatory mediators may be useful indicators of early and chronic metabolic changes in the joint. Previous studies showed that inflammatory mediators increase in the synovial fluid after ACL injury, with concentrations peaking early and decreasing over time $[68,69]$. A recent study showed that synovial fluid Creactive protein transiently increased following $\mathrm{ACL}$ injury, but decreased from the initial peak within 3 months. In contrast, no substantive changes in IL-1B levels were found among the study's 11 participants [70]. In another study, higher concentrations of IL-6, IFN $\gamma$, MIP-1 $\beta$, and MCP- 1 were measured in the synovial fluid of ACL-injured patients versus uninjured controls [71]. Synovial biomarkers from meniscusinjured populations, including both acute and chronic injury, had higher levels of cytokines IFN $\gamma$, IL-6, MCP-1, MIP-1 $\beta$, IL-2, IL-4, IL-10, and IL-13 compared with asymptomatic populations with IL-6, MCP-1, MIP-1 $\beta$, and IFNY concentrations correlating with patientreported pain outcome scores [72].
These studies demonstrate that inflammatory mediators transiently increase following acute ACL injury, with levels decreasing over time. The data from meniscusinjured subjects suggest persistence or resurgence of inflammation, especially in those with pain. These data support the need for continued longitudinal assessment of inflammatory mediators in joint-injured cohorts as potential biomarkers for diagnosis and staging of preosteoarthritic conditions.

\section{Collagen and bone metabolites}

Byproducts of articular cartilage and bone metabolism were some of the first biomarkers assessed [73]. Early studies focused on biochemical biomarkers in populations with radiographic OA, typically in patients with Kellgren-Lawrence score 2 or higher. Recent studies of the synovial fluid in subjects with ACLT show that elevations of C-terminal cross-linked telopeptide type I and type II collagen, $\mathrm{N}$-terminal telopeptides of type I collagen, and $\mathrm{C} 1,2 \mathrm{C}$ - all biomarkers of collagen turnover - peak several weeks after injury [70]. There was also an increase in biomarkers associated with bone resorption. In the same study, strong correlations were found between serum and synovial fluid concentrations of bone metabolism biomarkers [70]. This study has refined the potential utility of biochemical biomarkers of cartilage and bone metabolism in early OA; however, further studies are needed.

\section{Glycosaminoglycans and hyaluronic acid}

Proteoglycans are important articular cartilage matrix components considered sensitive to changes in cartilage homeostasis [74,75]. Glycosaminoglycan and aggrecan ARGS fragments have been found to be transiently elevated in the synovial fluid of ACL-injured patients $[76,77]$. Aggrecan fragment ARGS-SELE remains elevated for at least 2 years after injury [76]. Serum is easier to obtain than synovial fluid and is of particular interest in biomarker analysis for screening purposes and for longitudinal studies. In a recent study, the concentration of W6F (a chondroitin sulfate epitope) was higher in the serum of ACL-injured subjects compared with uninjured controls [78]. Appropriate analysis of serum biomarkers probably cannot be performed in isolation because the detected concentrations represent processes within the entire body, while synovial fluid is more reflective of metabolism within the affected joints. These studies show promise in the use of biochemical biomarkers, particularly synovial fluid analysis, to characterize joint changes following ACL and meniscus injury. Analyses of serum, urine, and synovial fluid from these cohorts in conjunction with advanced structural imaging have the potential to yield candidate biomarkers that can be used for characterization of other pre-OA states. 


\section{Conclusion}

$\mathrm{OA}$ is a prevalent, disabling disease that currently lacks disease-modifying treatments. Clinical OA typically develops over decades and is strongly associated with modifiable factors such as joint injury, obesity, and mechanical overload. The ability to detect pre-osteoarthritic changes prior to the onset of irreversible changes is crucial for understanding the disease process, identifying potential disease-modifying treatments, and evaluating the effectiveness of new therapies. Comprehensive longitudinal studies of joint-injured cohorts allow for characterization of the earliest stages of joint degeneration, thereby maximizing opportunities for development and validation of biochemical and imaging biomarkers and for early intervention. The validation of tools suitable for diagnosis and staging of joint injury and early degeneration is needed to support a paradigm shift towards early treatment of pre-osteoarthritic conditions as a strategy to prevent or delay the onset of classic OA.

This article is part of the series on Cutting edge research and advances in orthopaedic research, edited by Edward Schwarz and Jay Lieberman. Other articles in this series can be found at http://arthritis-research.com/series/othopaedics

\section{Abbreviations}

$\mathrm{ACL}$, anterior cruciate ligament; $\mathrm{ACLT}$, anterior cruciate ligament tear; $\mathrm{BML}$, bone marrow lesions; dGEMRIC, delayed gadolinium-enhanced magnetic resonance imaging of cartilage; IFN, interferon; IL, interleukin; MCP-1, monocyte chemotactic protein-1; MIP-1 $\beta$, macrophage inflammatory protein-1 beta; $\mathrm{MRI}$, magnetic resonance imaging; $\mathrm{OA}$, osteoarthritis; $\mathrm{OCT}$, optical coherence tomography; qMRI, quantitative magnetic resonance imaging; UTE-T2*, ultrashort echo-time enhanced T2*.

\section{Competing interests}

The authors declare that they have no competing interests.

\section{Acknowledgements}

The authors would like to thank Patricia Stepp for her assistance with this paper. The concepts presented in this manuscript received peer-reviewed funding from the National Institutes of Health: NIH R01 AR052784 (CRC), R01 AR051963 (CRC), U13 AR060692 (CRC), P60 AR054731 (CRC), and RC2 AR058929 (CRC). The authors also acknowledge the Albert Ferguson Endowed Chair (CRC), Department of Orthopaedic Surgery, University of Pittsburgh.

\section{Author details}

${ }^{1}$ Cartilage Restoration Center, Department of Orthopaedic Surgery, University of Pittsburgh, Biomedical Science Tower E1640, 200 Lothrop Street, PA 15261 USA. ${ }^{2}$ Department of Orthopaedic Surgery, University of Pittsburgh, 3471 Fifth Avenue, Suite 911, Pittsburgh, PA 15213, USA.

Published: 7 June 2012

\section{References}

1. United States Bone and Joint Decade: The Burden of Musculoskeletal Diseases in the United States. Rosemont, IL: American Academy of Orthopaedic Surgeons; 2008

2. Chitnavis J, Sinsheimer JS, Clipsham K, Loughlin J, Sykes B, Burge PD, Carr AJ: Genetic influences in end-stage osteoarthritis. Sibling risks of hip and knee replacement for idiopathic osteoarthritis. J Bone Joint Surg Br 1997, 79:660-664.

3. McDonnell SM, Sinsheimer J, Price AJ, Carr AJ: Genetic influences in the aetiology of anteromedial osteoarthritis of the knee. J Bone Joint Surg Br 2007, 89:901-903.
4. Ryder JJ, Garrison K, Song F, Hooper L, Skinner J, Loke Y, Loughlin J, Higgins JP, MacGregor AJ: Genetic associations in peripheral joint osteoarthritis and spinal degenerative disease: a systematic review. Ann Rheum Dis 2008, 67:584-591.

5. Evangelou E, Chapman K, Meulenbelt I, Karassa FB, Loughlin J, Carr A, Doherty M, Doherty S, Gomez-Reino JJ, Gonzalez A, Halldorsson BV, Hauksson VB, Hofman A, Hart DJ, Ikegawa S, Ingvarsson T, Jiang Q, Jonsdottir I, Jonsson H, Kerkhof HJM, Kloppenburg M, Lane NE, Li J, Lories RJ, van Meurs JBJ, Na"kki A, Nevitt MC, Rodriguez-Lopez J, Shi D, Slagboom E, et al.: Large-scale analysis of association between GDF5 and FRZB variants and osteoarthritis of the hip, knee, and hand. Arthritis Rheum 2009, 60:1710-1721

6. Chu CR, Beynnon BD, Buckwalter JA, Garrett WE, Jr, Katz JN, Rodeo SA, Spindler KP, Stanton RA: Closing the gap between bench and bedside research for early arthritis therapies (EARTH): report from the AOSSM/NIH U-13 Post-Joint Injury Osteoarthritis Conference II. Am J Sports Med 2011, 39:1569-1578.

7. Brown TD, Johnston RC, Saltzman CL, Marsh JL, Buckwalter JA: Posttraumatic osteoarthritis: a first estimate of incidence, prevalence, and burden of disease. J Orthop Trauma 2006, 20:739-744.

8. Gelber AC, Hochberg MC, Mead LA, Wang NY, Wigley FM, Klag MJ: Joint injury in young adults and risk for subsequent knee and hip osteoarthritis. Ann Intern Med 2000, 133:321-328.

9. Lohmander LS, Englund PM, Dahl LL, Roos EM: The long-term consequences of anterior cruciate ligament and meniscus injures: osteoarthritis. Am J Sports Med 2007, 35:1756-1769.

10. Altman R, Asch E, Bloch D, Bole G, Borenstein D, Brandt K, Christy W, Cooke TD, Greenwald R, Hochberg M, Howell D, Kaplan D, Koopman W, Longley III S, Mankin H, McShane DJ, Medsger T Jr, Meenan R, Mikkelsen W, Moskowitz R, Murphy W, Rothschild B, Segal M, Sokoloff L, Wolfe F: Development of criteria for the classification and reporting of osteoarthritis. Classification of osteoarthritis of the knee. Diagnostic and Therapeutic Criteria Committee of the American Rheumatism Association. Arthritis Rheum 1986, 29:1039-1049.

11. Services USDoHaH: Clinical Development Programs for Drugs, Devices, and Biological Products Intended for the Treatment of Osteoarthritis (OA). Rockville, MD: Center for Devices and Radiological Health; 1999.

12. Chu CR, Williams A, Tolliver D, Kwoh CK, Bruno S, 3rd, Irrgang JJ: Clinical optical coherence tomography of early articular cartilage degeneration in patients with degenerative meniscal tears. Arthritis Rheum 2010, 62:1412-1420

13. Kieser CW, Jackson RW: Severin Nordentoft: the first arthroscopist. Arthroscopy 2001, 17:532-535.

14. Outerbridge RE: The etiology of chondromalacia patellae. J Bone Joint Surg Br 1961, 43-B:752-757.

15. Chu CR, Lin D, Geisler JL, Chu CT, Fu FH, Pan YT: Arthroscopic microscopy of articular cartilage using optical coherence tomography. Am J Sports Med 2004, 32:699-709.

16. Drexler W, Stamper D, Jesser C, Li XD, Pitris C, Saunders K, Martin S, Lodge MB, Fujimoto JG, Brezinski ME: Correlation of collagen organization with polarization sensitive imaging of in vitro cartilage: implications for osteoarthritis. J Rheumato/ 2000, 28:1311-1318.

17. Chu CR, Izzo NJ, Irrgang JJ, Ferretti M, Studer RK: Clinical diagnosis of potentially treatable early articular cartilage degeneration using optical coherence tomography. J Biomed Optics 2007, 12:051703-1-6.

18. O'Malley MJ, Chu CR: Arthroscopic optical coherence tomography in diagnosis of early arthritis. Minimally Invasive Surgery 2011, 2011:1-6.

19. Bear DM, Szczodry M, Kramer S, Coyle CH, Smolinski P, Chu CR: Optical coherence tomography detection of subclinical traumatic cartilage injury. J Orthop Trauma 2010, 24:577-582.

20. Neogi T, Felson D, Niu J, Lynch J, Nevitt M, Guermazi A, Roemer F, Lewis CE, Wallace B, Zhang Y: Cartilage loss occurs in the same subregions as subchondral bone attrition: a within-knee subregion-matched approach from the Multicenter Osteoarthritis Study. Arthritis Rheum 2009, 61:1539-1544.

21. Felson DT, Lynch J, Guermazi A, Roemer FW, Niu J, McAlindon T, Nevitt MC: Comparison of BLOKS and WORMS scoring systems part II. Longitudinal assessment of knee MRIs for osteoarthritis and suggested approach based on their performance: data from the Osteoarthritis Initiative. Osteoarthritis Cartilage 2010, 18:1402-1407.

22. Peterfy CG, Schneider E, Nevitt M: The osteoarthritis initiative: report on the 
design rationale for the magnetic resonance imaging protocol for the knee. Osteoarthritis Cartilage 2008, 16:1433-1441.

23. Eckstein F, Maschek S, Wirth W, Hudelmaier M, Hitzl W, Wyman B, Nevitt M, Le Graverand MP: One year change of knee cartilage morphology in the first release of participants from the Osteoarthritis Initiative progression subcohort: association with sex, body mass index, symptoms and radiographic osteoarthritis status. Ann Rheum Dis 2009, 68:674-679.

24. Bredella MA, Tirman PF, Peterfy CG, Zarlingo M, Feller JF, Bost FW, Belzer JP, Wischer TK, Genant HK: Accuracy of T2-weighted fast spin-echo MR imaging with fat saturation in detecting cartilage defects in the knee: comparison with arthroscopy in 130 patients. AJR Am J Roentgenol 1999, 172:1073-1080.

25. Potter HG, Foo LF: Magnetic resonance imaging of articular cartilage: trauma, degeneration, and repair. Am J Sports Med 2006, 34:661-677.

26. Owman $H$, Tiderius CJ, Neuman P, Nyquist F, Dahlberg LE: Association between findings on delayed gadolinium-enhanced magnetic resonance imaging of cartilage and future knee osteoarthritis. Arthritis Rheum 2008, 58:1727-1730.

27. Roos EM, Dahlberg L: Positive effects of moderate exercise on glycosaminoglycan content in knee cartilage: a four-month, randomized, controlled trial in patients at risk of osteoarthritis. Arthritis Rheum 2005, 52:3507-3514

28. Tiderius $C J$, Olsson LE, Leander P, Ekberg O, Dahlberg L: Delayed gadoliniumenhanced MRI of cartilage (dGEMRIC) in early knee osteoarthritis. Magn Reson Med 2003, 49:488-492.

29. Hovis KK, Stehling C, Souza RB, Haughom BD, Baum T, Nevitt M, McCulloch C, Lynch JA, LinkTM: Physical activity is associated with magnetic resonance imaging-based knee cartilage T2 measurements in asymptomatic subjects with and those without osteoarthritis risk factors. Arthritis Rheum 2011, 63:2248-2256.

30. Li X, Benjamin Ma C, Link TM, Castillo DD, Blumenkrantz G, Lozano Carballido-Gamio J, Ries M, Majumdar S: In vivo T(1rho) and T(2) mapping of articular cartilage in osteoarthritis of the knee using 3 T MRI. Osteoarthritis Cartilage 2007, 15:789-797

31. Stahl R, Luke A, Li X, Carballido-Gamio J, Ma CB, Majumdar S, Link TM: T1rho, $\mathrm{T} 2$ and focal knee cartilage abnormalities in physically active and sedentary healthy subjects versus early OA patients - a 3.0-Tesla MRI study. Eur Radio/ 2009, 19:132-143.

32. Du J, Takahashi AM, Chung CB: Ultrashort TE spectroscopic imaging (UTESI): application to the imaging of short $\mathrm{T} 2$ relaxation tissues in the musculoskeletal system. J Magn Reson Imaging 2009, 29:412-421.

33. Williams A, Qian Y, Chu CR: UTE-T2* mapping of human articular cartilage in vivo: a repeatability assessment. Osteoarthritis Cartilage 2011, 19:84-88.

34. Williams A, Qian Y, Golla S, Chu CR: UTE-T2* mapping detects sub-clinical meniscus injury after anterior cruciate ligament tear. Osteoarthritis Cartilage 2012, 20:486-494

35. Bashir A, Gray ML, Hartke J, Burstein D: Nondestructive imaging of human cartilage glycosaminoglycan concentration by MRI. Magn Reson Med 1999, 41:857-865.

36. Williams A, Sharma L, McKenzie CA, Prasad PV, Burstein D: Delayed gadolinium-enhanced magnetic resonance imaging of cartilage in knee osteoarthritis: findings at different radiographic stages of disease and relationship to malalignment. Arthritis Rheum 2005, 52:3528-3535.

37. Dardzinski BJ, Mosher TJ, Li S, Van Slyke MA, Smith MB: Spatial variation of T2 in human articular cartilage. Radiology 1997, 205:546-550.

38. Goodwin DW, Wadghiri YZ, Zhu H, Vinton CJ, Smith ED, Dunn JF: Macroscopic structure of articular cartilage of the tibial plateau: influence of a characteristic matrix architecture on MRI appearance. AJRAm J Roentgenol 2004, 182:311-318.

39. Mosher TJ, Dardzinski BJ, Smith MB: Human articular cartilage: influence of aging and early symptomatic degeneration on the spatial variation of T2 - preliminary findings at 3 T. Radiology 2000, 214:259-266.

40. Menezes NM, Gray ML, Hartke JR, Burstein D: T2 and T1 rho MRI in articular cartilage systems. Magn Reson Med 2004, 51:503-509.

41. Williams A, Qian Y, Bear D, Chu CR: Assessing degeneration of human articular cartilage with ultra-short echo time (UTE) T2* mapping Osteoarthritis Cartilage 2010, 18:539-546.

42. Steinbach LS, Suh KJ: Bone marrow edema pattern around the knee on magnetic resonance imaging excluding acute traumatic lesions. Semin Musculoskelet Radiol 2011, 15:208-220

43. Oda H, Igarashi M, Sase H, Sase T, Yamamoto S: Bone bruise in magnetic resonance imaging strongly correlates with the production of joint effusion and with knee osteoarthritis. J Orthop Sci 2008, 13:7-15.

44. Zhao J, Li X, Bolbos RI, LinkTM, Majumdar S: Longitudinal assessment of bone marrow edema-like lesions and cartilage degeneration in osteoarthritis using 3 T MR T1rho quantification. Skeletal Radiol 2010 39:523-531.

45. Crema MD, Roemer FW, Zhu Y, Marra MD, Niu J, Zhang Y, Lynch JA, Javaid MK, Lewis CE, El-Khoury GY, Felson DT, Guermazi A: Subchondral cystlike lesions develop longitudinally in areas of bone marrow edema-like lesions in patients with or at risk for knee osteoarthritis: detection with MR imaging - the MOST study. Radiology 2010, 256:855-862.

46. Kai B, Mann SA, King C, Forster BB: Integrity of articular cartilage on T2 mapping associated with meniscal signal change. Eur J Radiol 2010, 79:421-427.

47. Low AK, Chia MR, Carmody DJ, Lucas P, Hale D: Clinical significance of intrasubstance meniscal lesions on MRI. J Med Imaging Radiat Oncol 2008, 52:227-230.

48. Roemer FW, Guermazi A, Hunter DJ, Niu J, Zhang Y, Englund M, Javaid MK, Lynch JA, Mohr A, Torner J, Lewis CE, Nevitt MC, Felson DT: The association of meniscal damage with joint effusion in persons without radiographic osteoarthritis: the Framingham and MOST osteoarthritis studies. Osteoarthritis Cartilage 2009, 17:748-753.

49. Haywood L, MCWilliams DF, Pearson Cl, Gill SE, Ganesan A, Wilson D, Walsh DA: Inflammation and angiogenesis in osteoarthritis. Arthritis Rheum 2003, 48:2173-2177.

50. Smith MD, Triantafillou S, Parker A, Youssef PP, Coleman M: Synovial membrane inflammation and cytokine production in patients with early osteoarthritis. J Rheumato/ 1997, 24:365-371.

51. Myers SL, Brandt KD, Ehlich JW, Braunstein EM, Shelbourne KD, Heck DA, Kalasinski LA: Synovial inflammation in patients with early osteoarthritis of the knee. J Rheumatol 1990, 17:1662-1669.

52. Scanzello CR, McKeon B, Swaim BH, DiCarlo E, Asomugha EU, Kanda V, Nair A Lee DM, Richmond JC, Katz JN, Crow MK, Goldring SR: Synovial inflammation in patients undergoing arthroscopic meniscectomy: molecular characterization and relationship to symptoms. Arthritis Rheum 2011, 63:391-400.

53. Baker K, Grainger A, Niu J, Clancy M, Guermazi A, Crema M, Hughes L, Buckwalter J, Wooley A, Nevitt M, Felson DT: Relation of synovitis to knee pain using contrast-enhanced MRIs. Ann Rheum Dis 2010, 69:1779-1783.

54. Roemer FW, Guermazi A, Felson DT, Niu J, Nevitt MC, Crema MD, Lynch JA Lewis CE, Torner J, Zhang Y: Presence of MRI-detected joint effusion and synovitis increases the risk of cartilage loss in knees without osteoarthritis at 30-month follow-up: the MOST study. Ann Rheum Dis 2011, 70:1804-1809.

55. Han CW, Chu CR, Adachi N, Usas A, Fu FH, Huard J, Pan Y: Analysis of rabbit articular cartilage repair after chondrocyte implantation using optical coherence tomography. Osteoarthritis Cartilage 2003, 11:111-121.

56. Ugryumova N, Jacobs J, Bonesi M, Matcher SJ: Novel optical imaging technique to determine the 3-D orientation of collagen fibers in cartilage: variable-incidence angle polarization-sensitive optical coherence tomography. Osteoarthritis Cartilage 2009, 17:33-42

57. Mlynarik V, Trattnig S, Huber M, Zembsch A, Imhof H: The role of relaxation times in monitoring proteoglycan depletion in articular cartilage. J Magn Reson Imaging 1999, 10:497-502.

58. Nieminen MT, Rieppo J, Silvennoinen J, Toyras J, Hakumaki JM, Hyttinen MM, Helminen HJ, Jurvelin JS: Spatial assessment of articular cartilage proteoglycans with Gd-DTPA-enhanced T1 imaging. Magn Reson Med 2002 48:640-648.

59. Akella SV, Regatte RR, Gougoutas AJ, Borthakur A, Shapiro EM, Kneeland JB, Leigh JS, Reddy R: Proteoglycan-induced changes in T1rho-relaxation of articular cartilage at 4T. Magn Reson Med 2001, 46:419-423.

60. Bear DM, Williams A, Chu CT, Coyle CH, Chu CR: Optical coherence tomography grading correlates with MRI T2 mapping and extracellular matrix content. J Orthopaedic Res 2009, 28:546-552.

61. Xia Y, Moody JB, Burton-Wurster N, Lust G: Quantitative in situ correlation between microscopic MRI and polarized light microscopy studies of articular cartilage. Osteoarthritis Cartilage 2001, 9:393-406.

62. Li W, Scheidegger R, Wu Y, Edelman RR, Farley M, Krishnan N, Burstein D, Prasad PV: Delayed contrast-enhanced MRI of cartilage: comparison of nonionic and ionic contrast agents. Magn Reson Med 2010, 64:1267-1273.

63. Henkelman RM, Stanisz GJ, Menezes N, Burstein D: Can MTR be used to 
assess cartilage in the presence of Gd-DTPA2-? Magn Reson Med 2002, 48:1081-1084.

64. Matyas JR, Atley L, lonescu M, Eyre DR, Poole AR: Analysis of cartilage biomarkers in the early phases of canine experimental osteoarthritis. Arthritis Rheum 2004, 50:543-552.

65. Heard BJ, Achari Y, Chung M, Shrive NG, Frank CB: Early joint tissue changes are highly correlated with a set of inflammatory and degradative synovial biomarkers after $\mathrm{ACL}$ autograft and its sham surgery in an ovine model. J Orthop Res 2011, 29:1185-1192.

66. Kraus VB: Osteoarthritis year 2010 in review: biochemical markers. Osteoarthritis Cartilage 2011, 19:346-353.

67. Patra D, Sandell LJ: Recent advances in biomarkers in osteoarthritis. Curr Opin Rheumatol 2011, 23:465-470.

68. Cameron ML, Fu FH, Paessler HH, Schneider M, Evans CH: Synovial fluid cytokine concentrations as possible prognostic indicators in the $\mathrm{ACL}$ deficient knee. Knee Surg Sports Traumatol Arthrosc 1994, 2:38-44.

69. Irie K, Uchiyama E, Iwaso H: Intraarticular inflammatory cytokines in acute anterior cruciate ligament injured knee. Knee 2003, 10:93-96.

70. Catterall JB, Stabler TV, Flannery CR, Kraus VB: Changes in serum and synovial fluid biomarkers after acute injury (NCT00332254). Arthritis Res Ther 2010, 12:R229.

71. Cuellar VG, Cuellar JM, Golish SR, Yeomans DC, Scuderi GJ: Cytokine profiling in acute anterior cruciate ligament injury. Arthroscopy 2010, 26:1296-1301.

72. Cuellar JM, Scuderi GJ, Cuellar VG, Golish SR, Yeomans DC: Diagnostic utility of cytokine biomarkers in the evaluation of acute knee pain. J Bone Joint Surg Am 2009, 91:2313-2320.

73. Hollander AP, Heathfield TF, Webber C, Iwata Y, Bourne R, Rorabeck C, Poole AR: Increased damage to type II collagen in osteoarthritic articular cartilage detected by a new immunoassay. J Clin Invest 1994, 93:1722-1732

74. Lohmander LS, lonescu M, Jugessur H, Poole AR: Changes in joint cartilage aggrecan after knee injury and in osteoarthritis. Arthritis Rheum 1999, 42:534-544.

75. Witter J, Roughley PJ, Webber C, Roberts N, Keystone E, Poole AR: The immunologic detection and characterization of cartilage proteoglycan degradation products in synovial fluids of patients with arthritis. Arthritis Rheum 1987, 30:519-529.

76. Struglics A, Hansson M, Lohmander LS: Human aggrecanase generated synovial fluid fragment levels are elevated directly after knee injuries due to proteolysis both in the inter globular and chondroitin sulfate domains. Osteoarthritis Cartilage 2011, 19:1047-1057.

77. Larsson S, Lohmander LS, Struglics A: Synovial fluid level of aggrecan ARGS fragments is a more sensitive marker of joint disease than glycosaminoglycan or aggrecan levels: a cross-sectional study. Arthritis Res Ther 2009, 11:R92.

78. Pruksakorn D, Rojanasthien S, Pothacharoen P, Luevitoonvechkij S, Wongtreratanachai $\mathrm{P}$, Ong-Chai $\mathrm{S}$, Kongtawelert $\mathrm{P}$ : Chondroitin sulfate epitope (WF6) and hyaluronic acid as serum markers of cartilage degeneration in patients following anterior cruciate ligament injury. J Sci Med Sport 2009, 12:445-448.

doi:10.1186/ar3845

Cite this article as: Chu CR, et al.: Early diagnosis to enable early treatment of pre-osteoarthritis. Arthritis Research \& Therapy 2012, 14:212 